\title{
The role of MT1-MMP in the progression and metastasis of osteosarcoma
}

\author{
Hannah L. M. Spencer, Steven D. Shnyder, Paul M. Loadman, Robert A. Falconer \\ Institute of Cancer Therapeutics, Faculty of Life Sciences, University of Bradford, Bradford BD7 1DP, UK. \\ Correspondence to: Prof. Robert A. Falconer, Institute of Cancer Therapeutics, Faculty of Life Sciences, University of Bradford, \\ BD7 1DP, UK. E-mail: r.a.falconer1@bradford.ac.uk

\begin{abstract}
How to cite this article: Spencer HLM, Shnyder SD, Loadman PM, Falconer RA. The role of MT1-MMP in the progression and metastasis of osteosarcoma. J Cancer Metastasis Treat 2022;8:2. https://dx.doi.org/10.20517/2394-4722.2021.174
\end{abstract}

Received: 6 Sep 2021 First Decision: 26 Oct 2021 Revised: 3 Nov 2021 Accepted: 16 Nov 2021 Published: 14 Jan 2022

Academic Editors: Lucio Miele, RE Coleman Copy Editor: Xi-Jun Chen Production Editor: Xi-Jun Chen

\begin{abstract}
The dysregulation of Membrane - type 1 matrix metalloproteinase (MT1-MMP) has been extensively studied in numerous cancer types, and plays key roles in angiogenesis, cancer progression, and metastasis. MT1-MMP is a predictor of poor prognosis in osteosarcoma (OS), yet the molecular mechanisms of disease progression are unclear. This review provides a summary of the literature relating to the gene and protein expression of MT1-MMP (MMP-14) in OS clinical samples, evaluates the expression in cell lines and experimental models, and analyses its potential role in the progression and metastasis of OS. In addition, the therapeutic potential of MT1-MMP as a drug target has been assessed. Due to the biological complexity of MMPs, inhibition has proven to be challenging. However, exploiting the expression and proteolytic capacity of MT1-MMP could open new avenues in the search for novel, safer and selective drugs for use in OS.
\end{abstract}

Keywords: Matrix metalloproteinase, membrane-type, osteosarcoma, MT1-MMP

\section{INTRODUCTION}

Osteosarcoma (OS) is the most common form of primary malignant bone tumour and exhibits its highest prevalence in children and adolescents, with $80 \%$ of cases affecting individuals before the age of 20 . OS accounts for less than $1 \%$ of all new cancer diagnoses with an age-standardised incidence rate of 0.9 per 100,000 in the UK, affecting 3.4 per million people worldwide each year. The survival rate for those who 
develop OS, especially for those who have developed metastases, has not significantly improved over the past 30 years, with only $55 \%$ of patients surviving for more than 10 years. This is primarily attributed to the lack of novel therapeutics ${ }^{[1,2]}$.

Matrix metalloproteinases (MMPs) are a family of zinc-dependent endopeptidases that play key roles in angiogenesis, cancer progression and metastasis. These proteases are classified based on their structural characteristics and are often subdivided into two major groups; secreted MMPs and membrane-type MMPs $(\mathrm{MT}-\mathrm{MMPs})^{[3]}$. In normal tissues, MMPs are involved in a variety of physiological processes, including mediation of inflammation and tissue remodelling. However, the overexpression of MMPs in cancer has been associated with poor prognosis, and they have been considered as potential diagnostic and prognostic biomarkers in several cancer types, including OS, as exemplified by MT1-MMP (see Figure 1). Technically speaking, MT1-MMP is the name for the protein, whereas MMP-14 refers to the gene, but these terms are often used interchangeably in the literature.

The activation of MT-MMPs, achieved through cleavage of a single protein and pro-peptide domain, promotes their transport to the cell surface where they degrade components of the extracellular matrix and basement membranes - either directly or indirectly through the recruitment of other MMPs - thereby facilitating invasion and metastasis ${ }^{[8]}$. In a variety of carcinomas, the dysregulation of MT1-MMP has been extensively studied, yet the molecular mechanisms underlying OS disease progression are unclear, but MT1MMP may play a key role ${ }^{[9]}$. Initially, this review will examine the expression of MT1-MMP in OS clinical tumour samples and cell lines, followed by a discussion as to the potential role of MT1-MMP in OS progression and metastasis, before finally considering opportunities for therapeutic targeting for the benefit of OS patients.

\section{EXPRESSION OF MT1-MMP IN OSTEOSARCOMA}

\section{In OS clinical samples}

The clinical expression of secreted MMPs, especially MMP-2 and MMP-9, has been the subject of many reviews. Both proteinases have been identified in tumour samples resected from patients with $\mathrm{OS}^{[10,11]}$. The differential expression of MT1-MMP in tumour tissue samples, when compared to normal tissues, has been widely researched; the data available for the expression of MT1-MMP in OS clinical tissues are summarised in this section.

Uchibori et al. ${ }^{[12]}$ examined 47 patient samples, all of which were taken prior to patients undergoing treatment who presented with no distant metastasis. MT1-MMP, MMP-2, -9, and tissue inhibitor of metalloproteinases 2 (TIMP-2) protein expression and their correlation with prognosis were examined immunohistochemically and by gelatin zymography. Strong expression (positive cell ratio $>50 \%$ ) of MT1MMP in 21 (45\%) samples was noted, 18 (38\%) of which were also positive for MMP-2 expression. The overall survival rate (OAS) for the 18 patients exhibiting strong expression of both MT1-MMP and MMP-2 was $57 \%$, whereas, for the other patients, OAS was $87 \%$. Although many patients within this study remained disease-free up until the follow-up (after 5 years), 15 patients developed metastasis, and 11 patients died of the disease. Localisation of MT1-MMP, MMP-2 and TIMP-2 to the cytoplasm and cell membrane was observed, with a significant association between the strong expression of MT1-MMP, but not MMP-2, and a reduction in patient survival when compared to tumour samples that exhibited weak expression. The eventfree survival rate (EFS) was determined for the surviving 36 patients, all of whom were in remission following neoadjuvant and adjuvant chemotherapy and surgical resection. The EFS at 5 years for those with tumours exhibiting strong MT1-MMP expression and for those with tumours exhibiting weak expression was $60 \%$ and $81 \%$, respectively. Of the 36 patients, 13 had tumours that exhibited strong expression of both 

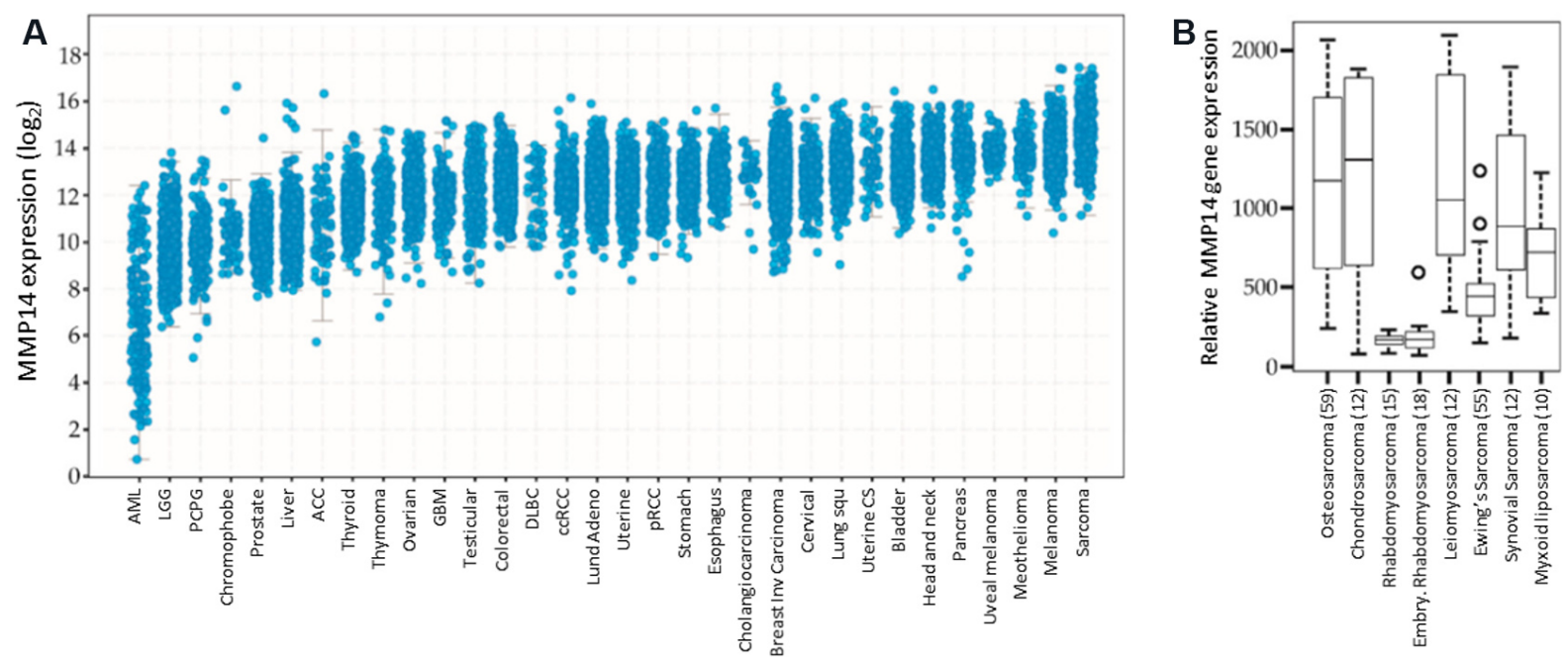

Figure 1. Expression of MT1-MMP in Sarcoma. (A) MMP-14 (the gene for MT1-MMP) expression in various cancer types (www.cbioportal.org/) $)^{[4,5]}$. (B) MMP-14 expression in various types of sarcoma (http://ist.medisapiens.com/) ${ }^{[6]}$. (Figure adapted from Figure 1 in $\left.\operatorname{Ref} 7^{[7]}\right)$.

MT1-MMP and MMP-2, which was also shown to correlate with poorer prognosis. In a follow-up study, it was found that the expression of extracellular matrix metalloproteinase inducer (EMMPRIN), a substrate of MT1-MMP which aids in MMP stimulation, and the co-expression of EMMPRIN and MT1-MMP were predictors of poor prognosis ${ }^{[13]}$.

In another study, the expression of MMPs and their endogenous inhibitors were investigated in 11 tumour xenografts and 12 OS biopsies (6 from primary tumours and 6 from metastases) ${ }^{[14]}$. MMP-14 mRNA, along with $M M P-2$, TIMP-1 and TIMP-2 mRNA, was identified in the majority of samples, with a consistent pattern of expression of $M M P-14, M M P-2$ and TIMP-2 in both the xenografts and biopsies. The authors stated that a pattern of MMP and TIMP expression in metastases, when compared with primary tumours, could not be discerned.

Utilising high-throughput sequencing, Xiao et al. ${ }^{[15]}$ screened for 339 genes known to be associated with cancer progression in 10 samples from OS patients from China. It was shown that the 10 samples tested demonstrated on average more than 200 gene mutations, including the $M M P-14$ gene. Although the findings from this study are in line with previously published studies ${ }^{[16]}$, a more comprehensive evaluation of the OS genome in larger collections of OS samples is required to identify high-yield mutations.

Whole transcriptosome analysis was performed on 33 OS patient samples. Tumour and normal tissues were collected from 18 bone samples following resection and 15 formalin-fixed paraffin-embedded (FFPE) samples that were retrieved from pathology archives. The genes that were most significantly downregulated and upregulated in the tumour tissues were identified ${ }^{[17]}$. MMP-14 was shown to be the 7 th most upregulated gene in osteosarcoma, with a greater than 2 -fold increase in gene expression in $87 \%$ of tumour samples when compared to their matched controls. Of these, $47 \%$ also showed elevated levels of $M M P-2$ and/or MMP-9. Enrichment analysis showed that there were significant alterations in the genes shown to regulate collagen degradation, extracellular matrix (ECM) organisation and ECM degradation, including collagens; COL11A1, COL2A1 and COL1OA1; among others. In the FFPE samples, it was evident that chemotherapy had not brought about any significant changes in $M M P-14, M M P-2$ and $M M P-9$ expression. 
A study by Engelholm et al. ${ }^{[18]}$ examined OS patient samples to identify novel bone degradation pathways. Of the 10 samples that were tested, by immunohistochemical analysis, for MT1-MMP protein expression, all were positive to varying degrees; scattered expression was observed in 2 samples, moderate expression $(<50 \%)$ in 4 samples and high $(>50 \%)$ expression in 4 samples. This study also demonstrated the differential expression of MMPs in primary OS tumours and tumours resulting from carcinomas. OS tumour samples showed high expression of MT1-MMP and other endocytic collagen receptors, whereas, in tumours originating from breast carcinoma, there was little to no expression of these proteins.

Also utilising immunostaining techniques, Biswas et al. ${ }^{[19]}$, demonstrated that in biopsies collected from patients that had undergone standard MAP chemotherapy - that involves administering high dose methotrexate $(\mathrm{M})$, doxorubicin (A - adriamycin) and cisplatin $(\mathrm{P})$ - more than $90 \%$ of samples evaluated expressed the MT1-MMP protein. A reduction in overall survival was associated with an increase in the localisation of MT1-MMP to the cytoplasm $(P=0.0002)$ and to the nucleus $(P<0.0001)$. In addition to this, a reduction in the EFS was observed in patients with localisation of MT1-MMP to the nucleus, but not to the cytoplasm. Therefore MT1-MMP localisation could prove to be crucial in predicting patient prognosis and in determining the patients' response chemotherapy.

\section{In experimental models of OS}

MMP expression in OS has garnered a lot of attention, but studies primarily focus on the expression of the widely-studied MMP-2 $2^{[1,20]}$ or MMP-9 ${ }^{[2,22]}$ rather than MT1-MMP (MMP-14). The vast majority of literature regarding gene expression of $M M P-14$ in human OS cell lines has been reported in $\mathrm{U}_{2} \mathrm{OS}$, Saos-2 and MG-63 cells, but there is some evidence of expression in OHS, HOS (HOS-MNNG and HOS-143B) and the newly derived HMOS cell lines (HMOS-A and HMOS-P) ${ }^{[23]}$. The phenotypical and functional characteristics of multiple osteosarcoma cell lines, both in vitro and in vivo, have been well documented. Of the cell lines examined, HOS cell lines, with the exception of HOS-MNNG, are highly tumorigenic and frequently form metastases (primarily in the lung), while U2OS, Soas-2 and MG-63 cells are less tumorigenic and non-metastatic ${ }^{[24,25]}$.

Giambernardi et al. ${ }^{[26]}$ reported the differential gene expression of MMPs in cell lines; a strong expression of $M M P-14$ was observed in HOS, MG-63 and Saos-2. It has been shown that the transformation of the HOSTe85 parental cell line to its 143B derivative, brought about an increase in MT1-MMP protein expression, which has been associated with a more metastatic phenotype, whereas a reduction in MT1-MMP expression in the transformed HOS-MNNG cell line played a role in reducing its metastatic capacity ${ }^{[27]}$.

Hypoxia, resulting from oxygen deprivation, is common in many solid tumours with an associated increase in hypoxia-related markers, such as hypoxia-inducible factors- $1 \alpha$ or $-2 \alpha$ (HIF-1 $\alpha /-2 \alpha$ ), which often correlates with poorer prognoses. Many genes, including those of MMPs, are transcriptionally regulated by these $\mathrm{HIFs}^{[28]}$. Chan et al. ${ }^{[29]}$ determined, by way of Western blot, that the degree of MT1-MMP protein expression in $\mathrm{U}_{2} \mathrm{OS}$ cells was equal to that of the positive control cell line HT1080, which exhibits a highlevel expression of the majority of MMPs. Further to this, immunohistochemical staining revealed that, in normoxia, MT1-MMP is primarily located in the cell cytoplasm and was shown to interact with HIF-1 $\alpha$ this interaction was not observed following a reduction in $\mathrm{O}_{2}$ levels. Hypoxia-induced alterations in the subcellular location of MT1-MMP, with a higher expression of MT1-MMP observed within the nucleus. In normoxia, a proximity ligation assay showed that there is minimal interaction between HIF- $2 \alpha$ and MT1MMP, but when cells were cultured under hypoxic conditions, there was a marked increase in the interaction signals within the nucleus. This interaction was shown to be specific to U2OS cells when compared to bone marrow mesenchymal stem cells. 
Transfection of $143 \mathrm{~B}$ cells, with a luciferase-Tomato reporter gene ( $p F U L T$ ) expressing firefly luciferase 2, allowed for the development of murine orthotopic models of OS that are easily traceable in vivo. Also, the cells were labelled with tdTomato making it possible to distinguish between tumour cells and host cells during immunohistochemical analysis. Histological characterisation of tissue samples generated from this orthotopic model revealed that they mimicked human disease, with a strong expression of MT1-MMP in tumour cells excised from the tibia and from metastatic lesions that had developed in the lungs. In addition to this, utilising Crispr/Cas9 gene editing techniques, the researchers generated MT1-MMP knockout and wildtype cells lines (also expressing luciferase and tdTomato), both of which were screened for MT1-MMP protein expression by western blot and genotyped. Comparison of these cell lines revealed that loss of MT1MMP expression resulted in a reduction in the ability of $143 \mathrm{~B}$ cells to degrade cellular collagen. Further investigation showed that there was no difference in the extent of bone degradation or the formation of lung metastases in orthotopic models of the newly derived cell lines, however. This suggests that MT1-MMP does not play a direct role in bone degradation or in the lung colonisation processes in 143B orthotopic models of OS, but rather acts via alternative $\operatorname{methods}^{[30]}$.

The differential expression of MT1-MMP in OS cells and clinical samples, when compared to controls, provides compelling evidence for its involvement in OS development. Many of the clinical studies are limited by small sample sizes, a common problem encountered when evaluating protein expression in OS tumours (given the rarity of the disease), and a more comprehensive study with a larger sample size could provide a better understanding of the role of MT1-MMP in OS. Within the tumour microenvironment, the complex network of pathways, many of which could be involved in the regulation of MT1-MMP, determines the rate of progression and metastasis in OS. These pathways, summarised in Figure 2, will be considered in the following section.

\section{ROLE OF MT1-MMP IN OS PROGRESSION AND METASTASIS}

The regulation of MT1-MMP expression by the key SRC/ERK1/2 signalling pathway (a major player in OS progression, Figure 2) in response to WNT5A has been investigated by Wang et al. ${ }^{[31]}$. They found that WNT5A-induced MG-63 cell migration and invasion was in part due to the activation of SRC/ERK1/2 signalling pathways that were shown to upregulate downstream MT1-MMP. Previous studies have reported that WNT5A-induced invasion in osteosarcoma cell lines (U2OS and Saos-2) is mediated by SRC through upregulation of MMP-13 $3^{[32,33]}$. The authors speculated that activation of the SRC/ERK1/2 signalling pathway is responsible for the increased expression of both MT1-MMP and MMP-13 in response to WNT5A.

The activity of a Wnt co-receptor, low-density lipoprotein receptor-related protein 5 (LRP5), has been linked with OS metastasis and poorer prognosis. Canonical Wnt signalling is responsible for the downstream regulation of a broad spectrum of cell cycle regulators, oncogenes and matrix-degrading enzymes. The transfection of a soluble form of LRP5 (sLRP5) into SaOS-2 cells significantly reduced the protein expression of MT1-MMP and MMP-2, with the effect on MMP-2 being more pronounced. MT1MMP is a known Wnt/ $\beta$-catenin target gene and membrane-bound activator of MMP- $2^{[34]}$. The authors did note that due to the differences in genetic backgrounds and mutational profiles of OS cell lines, the results here in Saos-2 cells cannot be generalised. Therefore further investigation of sLRP5 activity in additional cell lines that are stable following sLRP5 transfection is required ${ }^{[35]}$.

$\alpha 1$-Antitrypsin Portland ( $\alpha 1-\mathrm{PDX})$, a furin inhibitor, has been shown to affect the migration and invasion of MG-63 and Saos-2 OS cell lines ${ }^{[36]}$. This study suggested that $\alpha 1-P D X$ may play a key role in the inhibition of migration and invasion through the non-canonical Wnt pathway-mediated downregulation of MT1-MMP expression. It would be interesting to see what further exploration into the effect $\alpha 1$-PDX has on the activity 


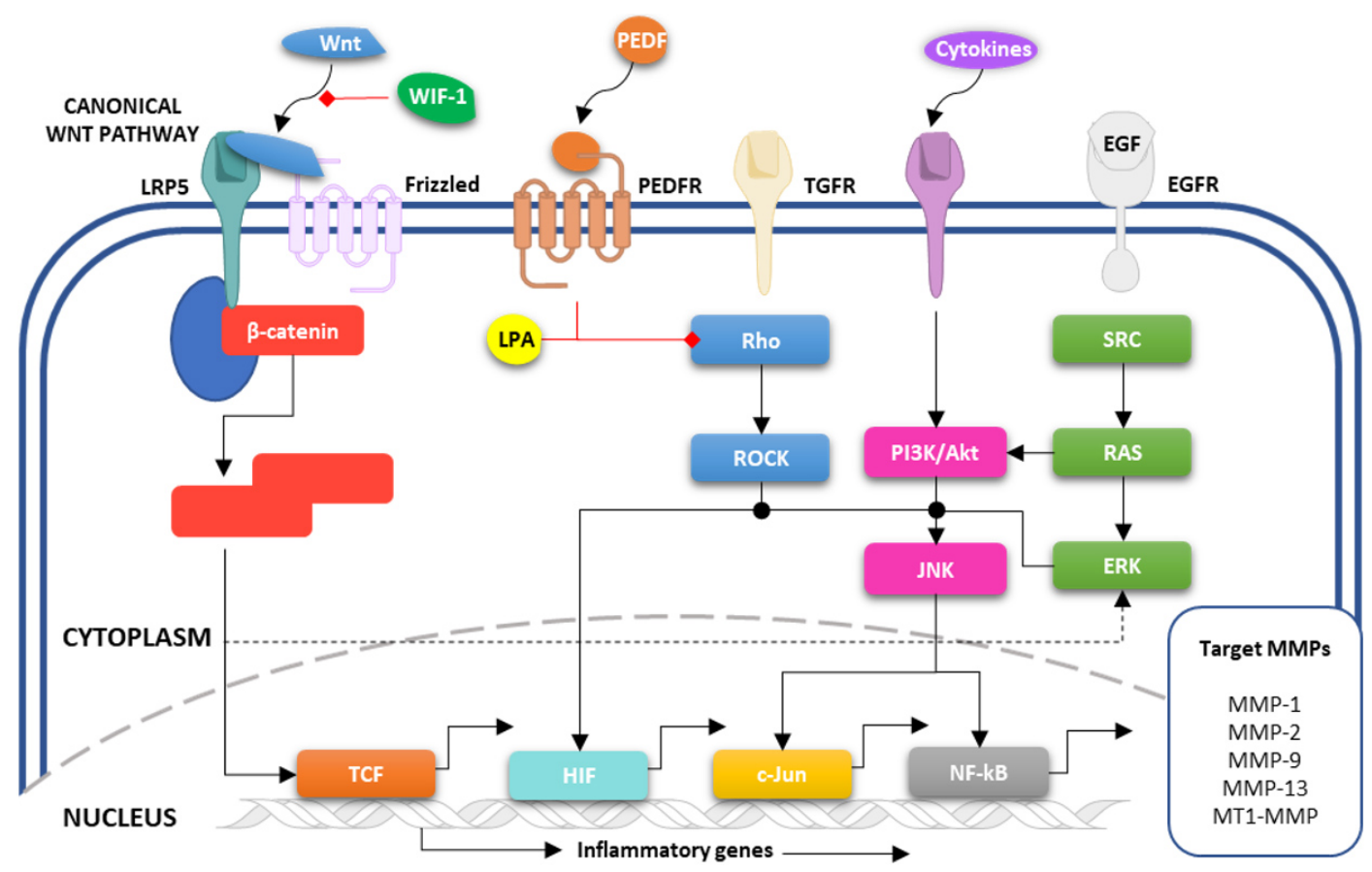

Figure 2. Schematic representation of potential MT1-MMP regulatory mechanisms in OS. Several growth factors (including Wnt, PEDF, TGF, EGF) activate downstream signal transduction pathways (including SRC, RAS, PI3K/Akt, Rho/ROCK) that are responsible for the transcriptional regulation of a variety of MMPs, including MT1-MMP. EGF: Epidermal growth factor; ERK: extracellular signal-regulated kinases; PEDF: pigment epithelium-derived factor; PI3K: phosphoinositide 3-kinases; ROCK: Rho-associated protein kinase; SRC: protooncogene tyrosine-protein kinase; TGF: transforming growth factor.

of other MMPs, especially MMP-2 and TIMPs reveals.

Wnt inhibitory factor 1 (WIF-1) protein levels are often downregulated in OS cell lines, yet the role of WIF-1 in disease progression is largely unknown ${ }^{[37]}$. WIF-1 expression has been shown to decrease tumorigenesis and metastasis in HOS-143B cells ${ }^{[38]}$. HOS-143B cell lines are highly aggressive and frequently metastasise to the lung in xenograft model ${ }^{[3,400]}$. HOS-143B cells transfected with WIF-1 exhibited reduced expression of MT1-MMP, and also MMP-2 and MMP-9, but there was no significant effect on proMMP-2. The authors concluded that the anti-metastatic effect of WIF-1 induction could be of clinical importance in OS patients shown to have aberrant Wnt signalling.

RNA interference techniques (siRNA) have also been used to determine the effect of knockdown of $\beta$ catenin on the invasion, migration and sensitivity to chemotherapeutics in OS cells $s^{[41]}$. In line with previous work in $\mathrm{U}_{2} \mathrm{OS}$ cells ${ }^{[42]}$, the authors were able to demonstrate that the motility and invasive ability of MG-63 cells were affected by the inhibition of $\beta$-catenin, which was attributed to the downregulation of MT1-MMP expression.

In chondrosarcoma, breast and prostate cancer cell lines, pigment-epithelium derived factor (PEDF) has been shown to downregulate MT1-MMP protein expression ${ }^{[43,44]}$. The mechanism by which PEDF reduces the metastatic capacity of OS cells through MMP regulatory pathways was examined in HOS-143B cells ${ }^{[45]}$. Investigation revealed that increases in the protein expression of PEDF correlated with an increase in MT1MMP and collagen I expression, yet a decrease in the levels of MMP-2 was observed. The authors speculated that the antagonistic activity of both PEDF and MT1-MMP would be necessary for bone remodeling, which could explain the differential regulation of MT1-MMP by PEDF across cell lines. The 
downregulation of OS metastasis in HOS-143B cells was attributed to a decrease in MMP-2 expression, which is not in line with previous studies in breast and prostate cancer cell lines ${ }^{[44]}$, with the authors noting that the decrease in MMP-2 seen could be attributed to an increase in MMP-2 secretion into the medium which went un-monitored in this study.

The aberrant expression of microRNAs (miRNAs) has been implicated in cancer progression ${ }^{[25]}$. MiRNAs regulate gene expression through the induction of mRNA degradation or by translational regression. The ability of miRNAs to simultaneously target multiple genes increases their likelihood of success as therapeutic agents. Xie et al. ${ }^{[46]}$, investigated the expression of miR-302b and the mechanism by which it promotes cell invasion and migration in OS cell lines. The overexpression of miR-146b-5p was shown to suppress cell proliferation and increase apoptosis in OS cell lines (HOS-143B and MG-63) ${ }^{[47]}$. Mimics of miR-302b were shown to suppress the mRNA and protein levels of MT1-MMP and MMP-2 through the targeting of the Runxz gene, while the treatment of cells with an miR-302b inhibitor produced the opposite effect.

Alternatively, Elenjord et al. ${ }^{[48]}$ utilised ribozyme-transfected cell clones of OHS to determine the effect of collagen 1 expression on MMP activity in the absence/presence of S100 calcium-binding protein A4 (S100A4). Previous work demonstrated that reducing S100A4 in OS cell lines, both in vitro and in vivo, reduced their invasive and metastatic capacity through downregulation of MMPs and TIMPs ${ }^{[49,50]}$. In addition, a study by Loennechen et al. ${ }^{[51]}$ showed that colchicine induced MT1-MMP expression in an S100A4-independent manner, with higher levels of MT1-MMP observed upon induction of microtubule rearrangements following exposure to colchicine, as observed in both control and transfected cell lines. The authors were able to demonstrate that activation of proMMP-2 by MT1-MMP was mediated by TIMP-1 in OHS cells (control pH $\beta-1$, II-11a and II-11b). Another study evaluated the effect of R1881, a synthetic androgen analogue, on the expression of several MMPs and their endogenous inhibitors in LNCaP (prostatic adenocarcinoma) and OHS cells. TIMP-2 was shown to be inhibited in a time-dependent manner in MT1-MMP deficient LNCaP cells, which also correlated with an increase in the levels of prostate-specific antigen. In these cells, the mRNA levels of other MMPs (MMP-1, -2, -9, MMP-14 and TIMP-1) were not detected. An elevation in the expression of MMP-14 mRNA was seen when LNCaP cells were co-cultured with OHS cells. In this co-culture, the proteolytic activity of the MT1-MMP/TIMP-2/MMP-2 complex was unaffected by R1881 suggesting that this complex may provide an androgen-independent route for the development of bone metastases from prostate carcinoma ${ }^{[52]}$.

Lysophosphatidic acid (LPA) plays an important role in tumour cell invasion by promoting ECM degradation through the regulation of downstream targets. Investigation of the effect that LPA has on Rho, Rho-associated protein kinase (ROCK), MMPs and TIMPs, revealed that following high-dose treatment with LPA mRNA and protein levels of Rho, ROCK, MT1-MMP and TIMP-2 were reduced. Interestingly low dose LPA treatment resulted in increased mRNA levels of these proteins. Selective inhibition of Rho prevented LPA-mediated reduction in MT1-MMP and TIMP-2. Matsumoto et al. ${ }^{[27]}$ speculated that the Rho-ROCK pathway might be involved in MMP-2 activation through regulating MT1-MMP and TIMP-2 levels.

MMPs also play an important role in inflammatory processes potentiating the activity of inflammatory proteins, which result in the activation of many downstream signalling pathways, including JNK/c-jun ${ }^{[53]}$. The ability of Dz13, a DNA enzyme, to downregulate c-Jun has been shown to have a direct effect on OS progression in HOS-143B and SJSA-1 cells and resulted in an increased doxorubicin sensitivity in Saos-2 cells ${ }^{[54-56]}$. Tan et al. ${ }^{[57]}$ investigated the relationship between Dz13 and MMP expression in OS. A dose- 
dependent decrease in MT1-MMP and MMP-2 expression was observed following exposure to Dz13 in SJSA-1 OS cells, and this is in line with previous studies that have also demonstrated that MMP-2 and MMP-9 expression is regulated by c-Jun ${ }^{[8,59]}$.

Bisphosphonates have been shown to reduce invasion and metastasis and potentiate the effects of conventional chemotherapy in $\mathrm{OS}^{[14,60,61]}$. Heikkilä et al. ${ }^{[62]}$ investigated the effect of clodronate on MT1MMP mediated activation of MMP-2 and showed that increasing the concentration of this bisphosphonate reduced MT1-MMP and MMP-2 mRNA and protein expression in MG-63 cells. Previously, it has been shown that alendronate reduced the secretion of MMP-2 and is able to induce apoptosis in OS, while risedronate reduces mRNA and protein levels of MMP-2 and MMP-9 in U2OS and Saos-2 cell lines ${ }^{[63,64]}$. Unfortunately, the effect of alendronate and risedronate on MT1-MMP levels were not assessed in these studies.

In summary, MT1-MMP plays multiple roles in OS, many of which go beyond the roles associated with tumour migration and invasion, which can be directly attributed to its proteolytic capacity and extracellular matrix degradation (see Table 1). There is still much to be learnt, but as we have discussed, MT1-MMP has a significant impact on multiple intracellular signalling pathways, all of which adds to its importance as a key player in OS progression. The potential for targeting MT1-MMP as a therapeutic strategy, and the challenges associated with it will now be considered.

\section{CHALLENGES AND FUTURE PROSPECTS}

There is considerable evidence (both clinical and experimental) to support the role of MT1-MMP in metastasis in several cancers (notably breast and prostate cancers), but this is not the case in $\mathrm{OS}^{[0,65,66]}$. This could be attributed to the rarity of OS, which makes accessing tissue samples for gene mapping and experimentation very difficult. Therefore, utilising the metastatic potential of HOS-MNNG and HOS-143B cell lines could provide a better model by which to study the involvement of MT1-MMP in OS metastasis. In the UK, the ICONIC project, funded by the Bone Cancer Research Trust, aims to create an OS patient registry, providing clinicians and researchers with better access to patient data and samples, thereby helping them to better understand the disease and develop novel treatments ${ }^{[67]}$. Similar registries also exist in the $\mathrm{USA}^{[68,69]}$.

The MAP chemotherapy regime remains the standard of care for localised $\operatorname{OS}^{[1,70]}$. It has long been understood that significant side effects are associated with the use of chemotherapeutic agents; this is especially true for HD-MTX. HD-MTX has many adverse side effects resulting from its toxicity, and so requires the administration of a rescue dose of leucovorin following its infusion ${ }^{[7]}$. In addition to this, studies have shown that in OS, the transport of MTX across the cell membrane is impaired, therefore necessitating the larger doses required to achieve a therapeutic effect ${ }^{[72]}$.

There is a pressing need for novel therapeutics for OS patients, especially for those with metastatic disease or tumour recurrence, both of which correlate with an unfavourable prognosis, and MT1-MMP is clearly a target worthy of attention. One approach has been the development of synthetic MMP inhibitors (MMPIs), but unfortunately, no molecule has successfully made it to the clinic. Many MMPIs failed to progress beyond Phase III clinical trials due to poor specificity, deregulation of TIMPs and the off-target toxicity that has resulted in suboptimal dosing recommendations. Novel molecular biology techniques have been utilised to rapidly detect the expression profiles of MMP genes in each patient, determine the potential efficacy of MMPIs and therefore take an individualised approach to treatment ${ }^{[73]}$. The recent advances in MMPIs have been extensively reviewed by Gregg Fields ${ }^{[74]}$, who highlighted that despite previous failures in clinical trials, 
Table 1. Summary of published data describing MT1-MMP expression in osteosarcoma clinical samples and experimental models

\begin{tabular}{|c|c|c|c|c|c|c|}
\hline & Target MMP/TIMP & Method & Sample size & Cell line & Key points & Ref. \\
\hline \multirow[t]{6}{*}{$\begin{array}{l}\text { Clinical } \\
\text { samples }\end{array}$} & $\begin{array}{l}\text { MT1-MMP, MMP-2, -9, } \\
\text { TIMP-2 }\end{array}$ & IHC, zymography & 47 & - & $\begin{array}{l}21 \text { of } 47 \text { samples exhibited strong expression of MT1-MMP. A reduction in OAS and } \\
\text { EFS was seen in those with higher degrees of MT1-MMP expression }\end{array}$ & [12] \\
\hline & $\begin{array}{l}\text { MMP-14, MMP-2, } \\
\text { TIMP-1, TIMP-2 }\end{array}$ & $\begin{array}{l}\text { Zymography, NB, } \\
\text { mRNA sequencing }\end{array}$ & $\begin{array}{l}23(11 \\
\text { xenografts, } 12 \\
\text { biopsies) }\end{array}$ & U2OS, OHS, Saos-2 & $\begin{array}{l}\text { A consistent pattern of expression of MMP-14, MMP-2 and TIMP-2 was observed in } \\
\text { both xenografts and biopsies }\end{array}$ & {$[14]$} \\
\hline & MMP-14 & $\begin{array}{l}\text { RT-PCR, genomic } \\
\text { sequencing }\end{array}$ & 10 & - & Mutations were identified in more than 200 genes, including in MMP-14 & [15] \\
\hline & MMP-2, $-9,-14$ & $\begin{array}{l}\text { Whole } \\
\text { transcriptosome } \\
\text { analysis }\end{array}$ & $\begin{array}{l}33 \text { ( } 18 \text { bone, } 15 \\
\text { FFPE) }\end{array}$ & - & $\begin{array}{l}5365 \text { genes were found to be differentially expressed in OS and normal tissues. MMP- } \\
14 \text { was amongst the most upregulated }\end{array}$ & {$[17]$} \\
\hline & MT1-MMP & $\begin{array}{l}\text { IHC, confocal } \\
\text { microscopy }\end{array}$ & 10 & - & All samples displayed varying levels of MT1-MMP expression & {$[18]$} \\
\hline & MT1-MMP & IHC & 76 & U2OS, SJSA-2 & $\begin{array}{l}\text { In OS tumours, MT1-MMP was found to be expressed in both the nucleus and } \\
\text { cytoplasm. Nuclear MT1-MMP was associated with decreased EFS }\end{array}$ & [19] \\
\hline \multirow[t]{12}{*}{$\begin{array}{l}\text { Experimental } \\
\text { models }\end{array}$} & $\begin{array}{l}\text { MMP-1, }-2,-3,-7,-8, \\
-11,-12,-13,-14,-15,-16\end{array}$ & RT-PCR & - & HOS, MG-63 and Saos-2 & HOS, MG-63 and Saos-2 displayed strong expression of MT1-MMP & {$[26]$} \\
\hline & MT1-MMP & $\begin{array}{l}\text { WB, IHC, confocal } \\
\text { microscopy }\end{array}$ & - & $\begin{array}{l}\text { HT1080, U2OS, MDA- } \\
\text { MB-231, MCF-7, PC, } \\
\text { LNCaP }\end{array}$ & $\begin{array}{l}\text { U2OS cells have high MT1-MMP expression. In hypoxia, MT1-MMP localises to the } \\
\text { nucleus }\end{array}$ & [29] \\
\hline & MT1-MMP & IHC, WB, Crispr/Cas9 & - & $\begin{array}{l}\text { 143B WT, } 143 \text { MT1- } \\
\text { MMP K/O, 143B } \\
\text { xenograft }\end{array}$ & $\begin{array}{l}\text { High expression of MT1-MMP was observed in xenograft tumour cells excised from } \\
\text { the tibia and from metastatic lesions in the lungs. Crispr/Cas9 knockout of MT1-mMP } \\
\text { reduced the ability of } 143 \mathrm{~B} \text { cells to degrade cellular collagen }\end{array}$ & {$[30]$} \\
\hline & MMP-13, MT1-MMP & WB, IF & - & U2OS and Saos-2 & $\begin{array}{l}\text { The activation of SRC/ERK1/2 signalling pathways was shown to upregulate } \\
\text { downstream MT1-MMP }\end{array}$ & [31] \\
\hline & MMP-2, MT1-MMP & WB, IF, RT-PCR & - & Saos-2 & Transfection of sLRP5 into Saos-2 cells significantly reduced MT1-MMP expression & {$[34,35]$} \\
\hline & MT1-MMP & RT-PCR, ChIP & - & MG-63 and Saos-2 OS & Furin inhibition reduced the migration and invasion of MG-63 and Saos-2 cells & {$[36]$} \\
\hline & $\begin{array}{l}\text { MMP-2, MMP-9, MT1- } \\
\text { MMP }\end{array}$ & RT-PCR, WB, IHC & - & HOS-143B & $\begin{array}{l}\text { HOS- } 143 \text { B cells transfected with WIF-1 exhibited reduced expression of MT1-MMP, } \\
\text { MMP- } 2 \text { and MMP-9 but not proMMP-2 }\end{array}$ & {$[38]$} \\
\hline & MT1-MMP & RT-PCR, WB & - & U2OS, MG-63 & $\begin{array}{l}\text { The motility and invasive ability of MG- } 63 \text { cells was affected by the inhibition of } \beta \text { - } \\
\text { catenin by way of MT1-MMP downregulation }\end{array}$ & {$[41,42]$} \\
\hline & MMP-2, MT1-MMP & $\mathrm{IHC}, \mathrm{IF}$ & - & HOS-143B & $\begin{array}{l}\text { Increased PEDF expression correlated with an increase in MT1-MMP and collagen I } \\
\text { expression, yet a decrease in the levels of MMP-2 }\end{array}$ & {$[45]$} \\
\hline & $\begin{array}{l}\text { MMP-2, -9, 13, MT1- } \\
\text { MMP }\end{array}$ & RT-PCR, WB & - & HOS-143B and MG-63 & $\begin{array}{l}\text { Mimics of miR-302b were shown to suppress the mRNA and protein levels of MT1- } \\
\text { MMP and MMP-2 through the targeting of the Runx } 2 \text { gene }\end{array}$ & {$[46]$} \\
\hline & $\begin{array}{l}\text { MMP-1, -2, MT1-MMP, } \\
\text { TIMP-1, -2 }\end{array}$ & NB, WB, zymography & - & $\begin{array}{l}\text { OHS cells (control pH } \beta \\
-1, \mathrm{II}-11 \mathrm{a} \text { and II-11b) }\end{array}$ & $\begin{array}{l}\text { Activation of proMMP- } 2 \text { by MT1-MMP was mediated by TIMP- } 1 \text { in OHS cells. } \\
\text { Reducing S10OA4 in OHS cells reduced their invasive and metastatic capacity } \\
\text { through downregulation of MMPs and TIMPs }\end{array}$ & {$[48-51]$} \\
\hline & MMP-2, MMP-14, & NB, ELISA, WB, & - & LNCaP/OHS co-culture & MT1-MMP/TIMP-2/MMP-2 complex may provide an androgen-independent route & [52] \\
\hline
\end{tabular}




$\begin{array}{ll}\text { TIMP-2 } & \text { zymography } \\ \text { MT1-MMP, TIMP-2 } & \begin{array}{l}\text { RT-PCR, ELISA, WB, } \\ \text { zymography, IF }\end{array} \\ \text { MT1-MMP, MMP-2 } & \\ \text { MT1-MMP, MMP-2 } & \end{array}$

MNNG

SJSA-1

MG-63 for the development of bone metastases

Inhibition of Rho prevented LPA-mediated reduction in MT1-MMP and TIMP-2

A dose-dependent decrease in MT1-MMP and MMP-2 expression was observed following exposure to Dz13

Increasing the concentration of clodronate reduced MT1-MMP and MMP-2 mRNA and protein expression in MG-63 cells

ChIP: Chromatin immunoprecipitation; ELISA: enzyme-linked immunoassay; IF: immunofluorescence; IHC: immunohistochemistry; MMP: matrix metalloproteinase; MT-MMP: membrane-type MMP; NB: Northern blotting; RT-PCR: reverse transcription polymerase chain reaction; WB: Western blotting.

which were attributed to a lack of understanding of the biological complexities of MMPs, there had been some recent clinical successes. MMPIs have wideranging applications, from the treatment of HER2-negative adenocarcinomas to diabetic foot ulcers. Doxycycline and minocycline, which are broad-spectrum MMPIs, have also shown promise in the treatment of patients with relapse-remitting multiple sclerosis. Both were shown to reduce brain lesions and serum levels of MMP-9 and were considered to be effective, safe and well-tolerated. A potent, selective MMPI could make a significant clinical impact on OS therapy.

An alternative approach is to exploit the proteolytic activity of MT1-MMP to activate a prodrug (a drug administered in an inactive form, which is subsequently activated at the target site) in the tumour microenvironment. By adopting this approach, there is potential to overcome the off-target toxicity seen in many chemotherapeutic agents like HD-MTX, since the drug would only be released in the tumour microenvironment, thereby sparing normal tissues of exposure to the drug, while simultaneously enhancing its therapeutic index.

Two such examples of MT1-MMP targeted agents are BT1718 and ICT2588 (see Figure 3), which are currently being evaluated in other cancers, but have significant potential for OS therapy. BT1718, which was developed by researchers at Bicycle Therapeutics Ltd, is currently in Phase I/II clinical trials for the treatment of advanced solid tumours (Clinical Trials. gov Identifier: NCT03486730). The drug conjugate is comprised of a bicyclic peptide covalently linked to DM1, a potent anti-tubulin agent. The peptide sequence has been designed to specifically bind to MT1-MMP through a disulphide linker - taking advantage of its expression profile in many solid tumour models (including various sarcomas, non-small cell lung cancer and triple-negative breast cancer) - thereby

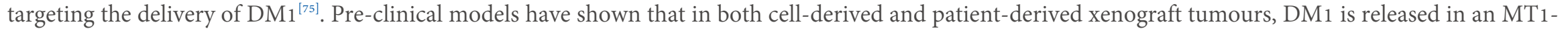
MMP dependent manner, which results in partial or complete tumour regression ${ }^{[76]}$. Although the clinical outcome is not yet known, this validates MT1-MMP as a target. ICT2588, which is currently being prepared for Phase I clinical trials, consists of 3 components; azademethylcolchicine, a vascular disrupting agent; an MT1-MMP activatable peptide sequence, Arg-Ser-Citrulline (Cit)-Gly-Homophenylalanine (Hof)-Tyr-Leu-Tyr; and a fluorescein isothiocyanate (FITC) based end cap. The release of the azademethylcolchicine warhead is achieved through (1) the selective cleavage of the peptide conjugate at the scissile bond between Gly and Hof; and (2) tumour exopeptidase activity removing the remaining amino acids. In tumour xenograft models, ICT2588 was shown to reduce tumour vasculature by up to $90 \%$ and, when co-administration with doxorubicin, led to a significant reduction in relative tumour volume ${ }^{[77]}$. Furthermore, 

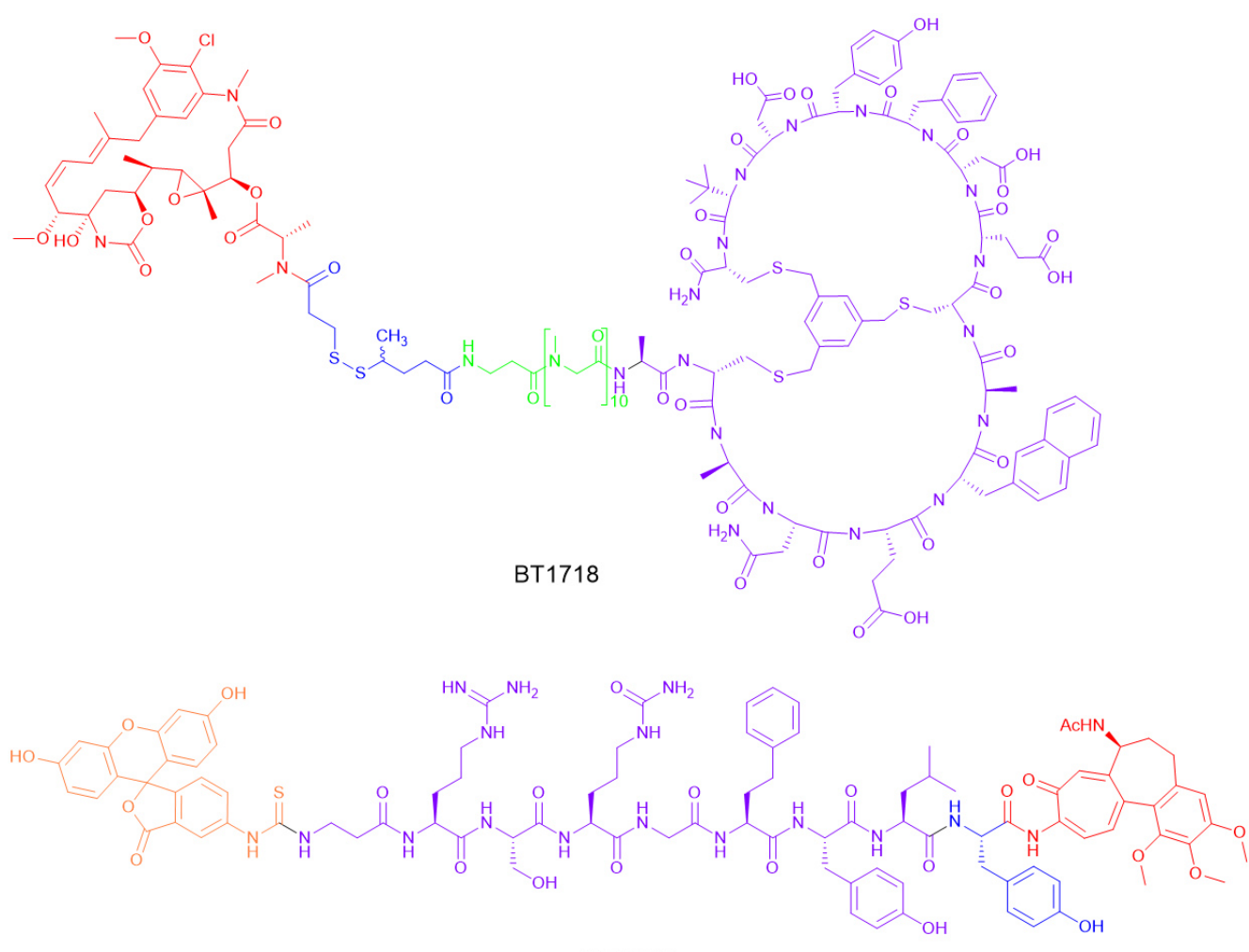

ICT2588

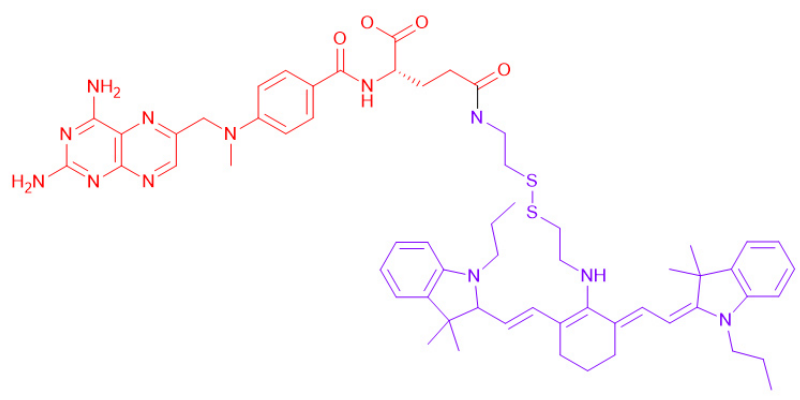

Cy-SS-MTX

Figure 3. Structures of BT1718, ICT2588 and Cy-SS-MTX.

ICT2588 was shown to lack the cardiotoxicity of the parent colchicine, achieved through selective targeting to the tumour ${ }^{[78]}$. Both of these approaches have significant potential application to OS, given the differential expression of MT1-MMP.

Alternative prodrug approaches to selectively deliver MTX have also been considered. For example, Cy-SSMTX (see Figure 3), is a glutathione-activated theranostic prodrug consisting of a cystamine functionalised IR780 (Cy), a near-infrared fluorescent heptamethine dye with tumour targeting properties, conjugated to MTX. In a co-culture of LO2 (normal hepatocyte) and MCF-7 (breast adenocarcinoma) cells, Cy-SS-MTX was shown to selectively accumulate within the mitochondria of MCF-7 cells. In addition, in both in vitro and in vivo models, Cy-SS-MTX was shown to reduce off-target toxicity and improve the anti-tumour efficiency of MTX ${ }^{[79]}$. 
In summary, after assessing the available evidence, it is clear that further scientific investigation is required to determine the full role that MT1-MMP plays in OS progression and metastasis. Its expression and proteolytic capacity are evident. However, MT1-MMP has significant therapeutic potential, particularly through the exploitation of its proteolytic activity, which allows for the selective release of toxic agents at the tumour site, helping to reduce off-target toxicity, which could drastically improve OS patient outcomes and quality-of-life.

\section{DECLARATIONS}

\section{Authors' contributions}

Wrote the manuscript: Spencer HLM

Concept: Falconer RA, Spencer HLM

Reviewed and edited manuscript: Spencer HLM, Shnyder SD, Loadman PM, Falconer RA

Final proofread: Spencer HLM, Falconer RA

\section{Availability of data and materials}

Not applicable.

\section{Financial support and sponsorship}

This work was supported by the Bone Cancer Research Trust (No. BCRT 6218).

\section{Conflicts of interest}

Falconer RA and Loadman PM declare that they are founding shareholders of Incanthera Plc. Other authors declared that there are no conflicts of interest.

\section{Ethical approval and consent to participate}

Not applicable.

\section{Consent for publication}

Not applicable.

\section{Copyright}

(c) The Author(s) 2022.

\section{REFERENCES}

1. Misaghi A, Goldin A, Awad M, Kulidjian AA. Osteosarcoma: a comprehensive review. SICOT J 2018;4:12. DOI PubMed PMC

2. UK CR. Bone sarcoma statistics. Available from: https://www.cancerresearchuk.org/ [Last accessed on 6 Dec 2021].

3. Visse R, Nagase H, Murphy G. Structure and function of matrix metalloproteinases and TIMPs. Cardiovasc Res 2006;69:562-73. DOI PubMed

4. Cerami E, Gao J, Dogrusoz U, et al. The cBio cancer genomics portal: an open platform for exploring multidimensional cancer genomics data. Cancer Discov 2012;2:401-4. DOI PubMed PMC

5. Gao J, Aksoy BA, Dogrusoz U, et al. Integrative analysis of complex cancer genomics and clinical profiles using the cBioPortal. Sci Signal 2013;6:p11. DOI PubMed PMC

6. Kilpinen S, Autio R, Ojala K, et al. Systematic bioinformatic analysis of expression levels of 17,330 human genes across 9,783 samples from 175 types of healthy and pathological tissues. Genome Biol 2008;9:R139. DOI PubMed PMC

7. Gonzalez-Molina J, Gramolelli S, Liao Z, Carlson JW, Ojala PM, Lehti K. MMP14 in sarcoma: a regulator of tumor microenvironment communication in connective tissues. Cells 2019;8:991. DOI PubMed PMC

8. Alaseem A, Alhazzani K, Dondapati P, Alobid S, Bishayee A, Rathinavelu A. Matrix metalloproteinases: a challenging paradigm of cancer management. Semin Cancer Biol 2019;56:100-15. DOI PubMed

9. Gong Y, Chippada-Venkata UD, Oh WK. Roles of matrix metalloproteinases and their natural inhibitors in prostate cancer progression. Cancers (Basel) 2014;6:1298-327. DOI PubMed PMC

10. Wang J, Shi Q, Yuan TX, et al. Matrix metalloproteinase 9 (MMP-9) in osteosarcoma: review and meta-analysis. Clin Chim Acta 2014;433:225-31. DOI PubMed

11. Zhang M, Zhang X. Association of MMP-2 expression and prognosis in osteosarcoma patients. Int J Clin Exp Pathol 2015;8:14965- 
70. PubMed PMC

12. Uchibori M, Nishida Y, Nagasaka T, Yamada Y, Nakanishi K, Ishiguro N. Increased expression of membrane-type matrix metalloproteinase-1 is correlated with poor prognosis in patients with osteosarcoma. Int J Oncol 2006. PubMed

13. Futamura N, Nishida Y, Urakawa H, et al. EMMPRIN co-expressed with matrix metalloproteinases predicts poor prognosis in patients with osteosarcoma. Tumour Biol 2014;35:5159-65. DOI PubMed

14. Bjørnland K, Flatmark K, Pettersen S, Aaasen AO, Fodstad O, Maelandsmo GM. Matrix metalloproteinases participate in osteosarcoma invasion. J Surg Res 2005;127:151-6. DOI PubMed

15. Xiao X, Wang W, Zhang H, et al. Individualized chemotherapy for osteosarcoma and identification of gene mutations in osteosarcoma. Tumour Biol 2015;36:2427-35. DOI PubMed

16. Choy E, Hornicek F, MacConaill L, et al. High-throughput genotyping in osteosarcoma identifies multiple mutations in phosphoinositide-3-kinase and other oncogenes. Cancer 2012;118:2905-14. DOI PubMed PMC

17. Ho XD, Phung P, Q Le V, et al. Whole transcriptome analysis identifies differentially regulated networks between osteosarcoma and normal bone samples. Exp Biol Med (Maywood) 2017;242:1802-11. DOI PubMed PMC

18. Engelholm LH, Melander MC, Hald A, et al. Targeting a novel bone degradation pathway in primary bone cancer by inactivation of the collagen receptor uPARAP/Endo180. J Pathol 2016;238:120-33. DOI PubMed

19. Biswas S, Rankin K, Long A, et al. Abstract 5580: Membrane-Type 1 Matrix Metalloproteinase (MT1-MMP): a predictive biomarker of chemotherapy response in osteosarcoma. Cancer Res 2012;72:5580. DOI

20. Wen X, Liu H, Yu K, Liu Y. Matrix metalloproteinase 2 expression and survival of patients with osteosarcoma: a meta-analysis. Tumour Biol 2014;35:845-8. DOI PubMed

21. Li H, Zhang K, Liu LH, et al. A systematic review of matrix metalloproteinase 9 as a biomarker of survival in patients with osteosarcoma. Tumour Biol 2014;35:5487-91. DOI PubMed

22. Liu Y, Wang Y, Teng Z, et al. Matrix metalloproteinase 9 expression and survival of patients with osteosarcoma: a meta-analysis. Eur J Cancer Care (Engl) 2017;26:e12364. DOI PubMed

23. Yamamoto Y, Yamamoto N, Tajima K, et al. Characterization of human multicentric osteosarcoma using newly established cells derived from multicentric osteosarcoma. J Cancer Res Clin Oncol 2011;137:423-33. DOI PubMed

24. Mohseny AB, Machado I, Cai Y, et al. Functional characterization of osteosarcoma cell lines provides representative models to study the human disease. Lab Invest 2011;91:1195-205. DOI PubMed

25. Lauvrak SU, Munthe E, Kresse SH, et al. Functional characterisation of osteosarcoma cell lines and identification of mRNAs and miRNAs associated with aggressive cancer phenotypes. Br J Cancer 2013;109:2228-36. DOI PubMed PMC

26. Giambernardi TA, Grant GM, Taylor GP, et al. Overview of matrix metalloproteinase expression in cultured human cells. Matrix Biol 1998;16:483-96. DOI PubMed

27. Matsumoto Y, Tanaka K, Harimaya K, Nakatani F, Matsuda S, Iwamoto Y. Small GTP-binding protein, Rho, both increased and decreased cellular motility, activation of matrix metalloproteinase 2 and invasion of human osteosarcoma cells. Jpn J Cancer Res 2001;92:429-38. DOI PubMed PMC

28. Petrova V, Annicchiarico-Petruzzelli M, Melino G, Amelio I. The hypoxic tumour microenvironment. Oncogenesis 2018;7:10. DOI PubMed PMC

29. Chan CD, Haagensen EJ, Tensaout HA, et al. Co-localisation of intra-nuclear membrane type-1 matrix metalloproteinase and hypoxia inducible factor-2 $\alpha$ in osteosarcoma and prostate carcinoma cells. Oncol Lett 2021;21:158. DOI PubMed PMC

30. Ingvarsen SZ, Gårdsvoll H, van Putten S, et al. Tumor cell MT1-MMP is dispensable for osteosarcoma tumor growth, bone degradation and lung metastasis. Sci Rep 2020;10:19138. DOI PubMed PMC

31. Wang X, Zhao X, Yi Z, et al. WNT5A promotes migration and invasion of human osteosarcoma cells via SRC/ERK/MMP-14 pathway. Cell Biol Int 2018;42:598-607. DOI PubMed

32. Enomoto M, Hayakawa S, Itsukushima S, et al. Autonomous regulation of osteosarcoma cell invasiveness by Wnt5a/Ror2 signaling. Oncogene 2009;28:3197-208. DOI PubMed

33. Ren D, Minami Y, Nishita M. Critical role of Wnt5a-Ror2 signaling in motility and invasiveness of carcinoma cells following Snailmediated epithelial-mesenchymal transition. Genes Cells 2011;16:304-15. DOI PubMed

34. Liu P, Yang J, Pei J, Pei D, Wilson MJ. Regulation of MT1-MMP activity by $\beta$-catenin in MDCK non-cancer and HT1080 cancer cells. J Cell Physiol 2010;225:810-21. DOI PubMed PMC

35. Guo Y, Zi X, Koontz Z, et al. Blocking Wnt/LRP5 signaling by a soluble receptor modulates the epithelial to mesenchymal transition and suppresses met and metalloproteinases in osteosarcoma Saos-2 cells. J Orthop Res 2007;25:964-71. DOI PubMed

36. Liu B, Li G, Wang X, Liu Y. A furin inhibitor downregulates osteosarcoma cell migration by downregulating the expression levels of MT1-MMP via the Wnt signaling pathway. Oncol Lett 2014;7:1033-8. DOI PubMed PMC

37. Lin CH, Ji T, Chen C, Hoang BH. Wnt signaling in osteosarcoma. In: Kleinerman MDE, editor. Current advances in osteosarcoma. Cham: Springer International Publishing; 2014. p. 33-45. DOI

38. Rubin EM, Guo Y, Tu K, Xie J, Zi X, Hoang BH. Wnt inhibitory factor 1 decreases tumorigenesis and metastasis in osteosarcoma. Mol Cancer Ther 2010;9:731-41. DOI PubMed PMC

39. Tsuge M, Osaki M, Sasaki R, Hirahata M, Okada F. SK-216, a novel inhibitor of plasminogen activator inhibitor-1, suppresses lung metastasis of human osteosarcoma. Int J Mol Sci 2018;19:736. DOI PubMed PMC

40. Brennecke P, Arlt MJ, Campanile C, et al. CXCR4 antibody treatment suppresses metastatic spread to the lung of intratibial human osteosarcoma xenografts in mice. Clin Exp Metastasis 2014;31:339-49. DOI PubMed PMC

41. Zhang F, Chen A, Chen J, Yu T, Guo F. SiRNA-mediated silencing of beta-catenin suppresses invasion and chemosensitivity to 
doxorubicin in MG-63 osteosarcoma cells. Asian Pac J Cancer Prev 2011;12:239-45. PubMed

42. Zhang F, Chen A, Chen J, Yu T, Guo F. Influence of $\beta$-catenin small interfering RNA on human osteosarcoma cells. J Huazhong Univ Sci Technolog Med Sci 2011;31:353-8. DOI PubMed

43. Ladhani O, Sánchez-Martinez C, Orgaz JL, Jimenez B, Volpert OV. Pigment epithelium-derived factor blocks tumor extravasation by suppressing amoeboid morphology and mesenchymal proteolysis. Neoplasia 2011;13:633-42. DOI PubMed PMC

44. Tan ML, Choong PF, Dass CR. Anti-chondrosarcoma effects of PEDF mediated via molecules important to apoptosis, cell cycling, adhesion and invasion. Biochem Biophys Res Commun 2010;398:613-8. DOI PubMed

45. Alcantara MB, Nemazannikova N, Elahy M, Dass CR. Pigment epithelium-derived factor upregulates collagen I and downregulates matrix metalloproteinase 2 in osteosarcoma cells, and colocalises to collagen I and heat shock protein 47 in fetal and adult bone. $J$ Pharm Pharmacol 2014;66:1586-92. DOI PubMed

46. Xie Y, Sun W, Deng Z, Zhu X, Hu C, Cai L. MiR-302b suppresses osteosarcoma cell migration and invasion by targeting Runx2. Sci Rep 2017;7:13388. DOI PubMed PMC

47. Xu E, Zhao J, Ma J, et al. miR-146b-5p promotes invasion and metastasis contributing to chemoresistance in osteosarcoma by targeting zinc and ring finger 3. Oncol Rep 2016;35:275-83. DOI PubMed

48. Elenjord R, Allen JB, Johansen HT, et al. Collagen I regulates matrix metalloproteinase-2 activation in osteosarcoma cells independent of S100A4. FEBS J 2009;276:5275-86. DOI PubMed

49. Bjørnland K, Winberg JO, Odegaard OT, et al. S100A4 involvement in metastasis: deregulation of matrix metalloproteinases and tissue inhibitors of matrix metalloproteinases in osteosarcoma cells transfected with an anti-S100A4 ribozyme. Cancer Res 1999;59:4702. PubMed

50. Mathisen B, Lindstad RI, Hansen J, et al. S100A4 regulates membrane induced activation of matrix metalloproteinase-2 in osteosarcoma cells. Clin Exp Metastasis 2003;20:701-11. DOI PubMed

51. Loennechen T, Mathisen B, Hansen J, et al. Colchicine induces membrane-associated activation of matrix metalloproteinase-2 in osteosarcoma cells in an S100A4-independent manner. Biochem Pharmacol 2003;66:2341-53. DOI PubMed

52. Bratland Å, Ragnhildstveit E, Bjørnland K, et al. The metalloproteinase inhibitor TIMP-2 is down-regulated by androgens in LNCaP prostate carcinoma cells. Clin Exp Metastasis 2003;20:541-7. DOI PubMed

53. Parks WC, Wilson CL, López-Boado YS. Matrix metalloproteinases as modulators of inflammation and innate immunity. Nat Rev Immunol 2004;4:617-29. DOI PubMed

54. Dass CR, Khachigian LM, Choong PF. c-Jun knockdown sensitizes osteosarcoma to doxorubicin. Mol Cancer Ther 2008;7:1909-12. DOI PubMed

55. Dass CR, Friedhuber AM, Khachigian LM, Dunstan DE, Choong PF. Downregulation of c-jun results in apoptosis-mediated antiosteosarcoma activity in an orthotopic model. Cancer Biol Ther 2008;7:1033-6. DOI PubMed

56. Dass CR, Tan ML, Galloway SJ, Choong PF. Dz13 induces a cytotoxic stress response with upregulation of E2F1 in tumor cells metastasizing to or from bone. Oligonucleotides 2010;20:79-91. DOI PubMed

57. Tan ML, Choong PF, Dass CR. Direct anti-metastatic efficacy by the DNA enzyme Dz13 and downregulated MMP-2, MMP-9 and MT1-MMP in tumours. Cancer Cell Int 2010;10:9. DOI PubMed PMC

58. Zhang G, Luo X, Sumithran E, et al. Squamous cell carcinoma growth in mice and in culture is regulated by c-Jun and its control of matrix metalloproteinase-2 and -9 expression. Oncogene 2006;25:7260-6. DOI PubMed

59. Fromigué O, Hamidouche Z, Marie PJ. Blockade of the RhoA-JNK-c-Jun-MMP2 cascade by atorvastatin reduces osteosarcoma cell invasion. J Biol Chem 2008;283:30549-56. DOI PubMed PMC

60. Tsubaki M, Satou T, Itoh T, et al. Reduction of metastasis, cell invasion, and adhesion in mouse osteosarcoma by YM529/ONO-5920induced blockade of the Ras/MEK/ERK and Ras/PI3K/Akt pathway. Toxicol Appl Pharmacol 2012;259:402-10. DOI PubMed

61. Meyers PA, Healey JH, Chou AJ, et al. Addition of pamidronate to chemotherapy for the treatment of osteosarcoma. Cancer 2011;117:1736-44. DOI PubMed PMC

62. Heikkilä P, Teronen O, Hirn MY, et al. Inhibition of matrix metalloproteinase-14 in osteosarcoma cells by clodronate. J Surg Res 2003;111:45-52. DOI PubMed

63. Cheng YY, Huang L, Lee KM, Li K, Kumta SM. Alendronate regulates cell invasion and MMP-2 secretion in human osteosarcoma cell lines. Pediatr Blood Cancer 2004;42:410-5. DOI PubMed

64. Xin ZF, Kim YK, Jung ST. Risedronate inhibits human osteosarcoma cell invasion. J Exp Clin Cancer Res 2009;28:105. DOI PubMed PMC

65. Linder S. MT1-MMP: Endosomal delivery drives breast cancer metastasis. J Cell Biol 2015;211:215-7. DOI PubMed PMC

66. Sheehy S, Annabi B. A transcriptional regulatory role for the membrane type-1 matrix metalloproteinase in carcinogen-induced inflammasome gene expression. Gene Regul Syst Bio 2017;11:1177625017713996. DOI PubMed PMC

67. ICONIC: Improving outcomes through collaboration in osteosarcoma. Available from: https://ClinicalTrials.gov/show/NCT04132895 [Last accessed on 6 Dec 2021].

68. Biology of osteosarcoma (BOOST) registry and biobank. Available from: https://clinicaltrials.gov/ct2/show/NCT03225872 [Last accessed on 6 Dec 2021].

69. Beech T. Patient/parent osteosarcoma genome-wide registry (POWR) provides the sarcoma community with an underutilized resource for genomic and medical history data. Available from: https://becauseofdaniel.org/the-science-of-powr/ [Last accessed on 6 Dec 2021].

70. Gill J, Ahluwalia MK, Geller D, Gorlick R. New targets and approaches in osteosarcoma. Pharmacol Ther 2013;137:89-99. DOI PubMed

71. Howard SC, McCormick J, Pui CH, Buddington RK, Harvey RD. Preventing and managing toxicities of high-dose methotrexate. 
Oncologist 2016;21:1471-82. DOI PubMed PMC

72. Sowers R, Wenzel BD, Richardson C, et al. Impairment of methotrexate transport is common in osteosarcoma tumor samples. Sarcoma 2011;2011:834170. DOI PubMed PMC

73. Winer A, Adams S, Mignatti P. Matrix metalloproteinase inhibitors in cancer therapy: turning past failures into future successes. Mol Cancer Ther 2018;17:1147-55. DOI PubMed PMC

74. Fields GB. The Rebirth of matrix metalloproteinase inhibitors: moving beyond the dogma. Cells 2019;8:984. DOI PubMed PMC

75. BT1718 in patients with advanced solid tumours. Available from: https://ClinicalTrials.gov/show/NCT03486730 [Last accessed on 6 Dec 2021].

76. Bennett G, Rigby M, Lutz B, Park P, Keen N. Abstract B135: The mechanism of action of BT1718, a novel small-molecule drug conjugate for the treatment of solid tumors expressing MT1-MMP. Mol Cancer Ther 2018;17:B135. DOI

77. Atkinson JM, Falconer RA, Edwards DR, et al. Development of a novel tumor-targeted vascular disrupting agent activated by membrane-type matrix metalloproteinases. Cancer Res 2010;70:6902-12. DOI PubMed PMC

78. Gill JH, Loadman PM, Shnyder SD, et al. Tumor-targeted prodrug ICT2588 demonstrates therapeutic activity against solid tumors and reduced potential for cardiovascular toxicity. Mol Pharm 2014;11:1294-300. DOI PubMed

79. Li S, Sun Z, Meng X, et al. Targeted methotrexate prodrug conjugated with heptamethine cyanine dye improving chemotherapy and monitoring itself activating by dual-modal imaging. Front Mater 2018;5:35. DOI 